\title{
DISTRIBUTIONS GAUSSIENNES ET TEMPS LOCAUX D'INTERSECTION
}

\author{
H.OUERDIANE et A. REZGUI
}

\begin{abstract}
Let $(\Omega, \mathcal{F}, \mathbb{P})$ be a probability space and $\left\{X_{t}, t \geq 0\right\}$ a stochastic gaussian process. Using white noise tools we give a mathematical meaning to this formel expression, $\int_{0}^{1} \int_{0}^{1} \delta\left(X_{t}-X_{s}\right) d s d t$, as a Hida distribution, this to exprime the intersection local time of any gaussian process.

As an example we study Brownian motion and Ornstein-Uhlenbeck process.
\end{abstract}

\section{Introduction}

Soit $(\Omega, \mathcal{F}, \mathbb{P})$ un espace probabilisé et $\left\{X_{t} ; t \geq 0\right\}$ un processus stochastique continu à valeur dans $\mathbf{R}^{d}, d \geq 1$. On définit pour $B$ borélien borné de $\mathbf{R}_{+}^{2}$ et pour presque tout $\omega \in \Omega$, la mesure $\mu_{B}$ de $\mathbf{R}^{d}$, dite mesure du temps local, par :

$$
\mu_{B}(\Gamma)=\lambda_{2}\left\{(t, s) \in B ; X_{t}-X_{s} \in \Gamma\right\}
$$

où $\Gamma$ est un borélien de $\mathbf{R}^{d}$ et où $\lambda_{2}$ est la mesure de Lebesgue de $\mathbf{R}_{+}^{2}$. Si de plus pour presque tout $\omega \in \Omega, \mu_{B}$ est absolument continue par rapport à la mesure de Lebesgue $\lambda_{d}$ de $\mathbf{R}^{d}$, on définit alors le temps local du processus $\left\{X_{t}\right\}$ par:

$$
\alpha(x, B):=\frac{d \mu_{B}}{d \lambda_{d}}(x) .
$$

Il se trouve alors que l'existence d'un tel temps local dépend non seulement du processus $\left\{X_{t}\right\}$ mais aussi de la dimension $d$.

Par exemple si $\left\{X_{t}\right\}$ est un mouvement Brownien, le temps local n'existe que pour $d=1,2$ et 3 voir [12],[2].

1991 Mathematics Subject Classification: 60G15-60G57-41-46.

Servicio de Publicaciones. Universidad Complutense. Madrid, 2000 
D'autre part le temps local $\alpha(x, B)$ exprime intuitivement le temps que le processus $\left\{X_{t}-X_{s}\right\}_{t, s \geq 0}$ passe au point $x$, pendant la durée $B$, ce qui nous permet de donner un sens à l'expression informelle " $\int_{B} \delta_{x}\left(X_{t}-X_{s}\right) d s d t "$ par $\alpha(x, B)$, où $\delta_{x}$ désigne la mesure de Dirac sur $\mathbf{R}^{d}$, au point $x$.

Lorsque $x=0, \int_{B} \delta_{0}\left(X_{t}-X_{s}\right) d s d t$ exprime donc le temps que la trajectoire $\left\{X_{t}(\omega)\right\}_{t \geq 0}$ passe en se rencontrant elle même et ceci pendant la durée $B$. Cette quantité, dite temps local d'intersection du processus $\left\{X_{t}\right\}$, a fait l'objet d'étude de plusieurs auteurs surtout dans le cas où le processus $\left\{X_{t}\right\}$ est le mouvement Brownien multidimensionel $\left\{B_{t}\right\}$. A partir de maintenant on note $\delta_{0}$ par $\delta$.

S.Varadhan dans [13], traite le cas $d=2$ et montre que:

$$
\int_{\Delta_{2}} \delta\left(B_{t}-B_{s}\right) d s d t=\alpha\left(0, \Delta_{2}\right)=+\infty P \text { - presque partout }
$$

où $\Delta_{2}=\left\{(t, s) \in[0,1]^{2} ; s<t\right\}$.

Néanmoins, il trouve une suite $\left(C_{k}\right)_{k \geq 0}$ de $\mathbf{R}_{+}$tel que

$$
\int_{\Delta_{2}} g_{k}\left(B_{t}-B_{s}\right) d s d t-C_{k}
$$

converge dans $L^{2}(\Omega, I \mathbb{P})$ vers une limite non nulle et où les fonctions $g_{k}$ sont définies par:

$$
g_{k}(x)=\frac{k}{2 \pi} e^{-k \frac{|x|^{2}}{2}}
$$

La suite de mesure $m_{k}=g_{k} d x$ converge étroitement vers la mesure de Dirac $\delta$.

J.F.Le Gall, retrouve dans [7] la renormalisation (2) de S.Varadhan. Plus précisément il détermine explicitement la suite $\left(C_{k}\right)$ ci-dessus et montre que:

$$
\int_{\Delta_{2}} \delta_{x}\left(B_{t}-B_{s}\right) d s d t-\frac{1}{2 \pi} \log \frac{1}{|x|}
$$

converge dans $L^{2}(\Omega, \mathbb{P})$ et $\mathbb{P}$-presque partout quand $x \rightarrow 0$ (toujours dans le cas $d=2$ ).

M.Yor dans [15], démontre dans le cas $d=3$ que

$$
\frac{1}{\sqrt{\log \frac{1}{|y|}}}\left\{2 \pi \int_{0}^{t} \int_{s}^{t} \delta_{y}\left(B_{u}-B_{s}\right) d s d u-\frac{t}{|y|}\right\}
$$


converge en loi vers le mouvement Brownien unidimensionel $\left(\beta_{t}\right)_{t \geq 0}$ indépendant de $\left(B_{t}\right)_{t \geq 0}$.

$H$. Watanabe dans [14], utilise une approche complétement différente. En effet il considère l'espace du bruit blanc $\left(S^{\prime}(\mathbb{R}), \gamma\right)$ où le mouvement Brownien unidimensionnel s'écrit $B_{t}(\omega)=\left\langle\omega, 1_{[0, t]}\right\rangle$, puis démontre que $L=\int_{\Delta_{2}} \delta\left(B_{t}-B_{s}\right) d s d t$ est un élément de $(S)^{*}$ l'espace de distributions de Hida [4][8].

Dans [1], M.Faria, T. Hida, L. Streit, H. Watanabe généralisent cette même approche pour étudier le cas du mouvement Brownien multidimensionnel et ils démontrent un théorème qui donne le lien entre la dimension $d \geq 1$ et le nombre $N$ de chaos qu'il faut retrancher de $L=\int_{\Delta_{2}} \delta\left(B_{t}-B_{s}\right) d s d t$ pour que l'expression obtenue notée $L^{(N)}$ ait un sens comme étant un élément de $(S)^{*}$.

Dans ce travail on commence par généraliser les résultats obtenus dans [1] en remplaçant le processus du mouvement Brownien $\left\{B_{t}\right\}$, qui s'écrit comme fonctionnelle du bruit blanc

$$
B_{t}(\omega)=\left(\left\langle\omega_{1}, \mathbf{1}_{[0, t]}\right\rangle, \cdots,\left\langle\omega_{d}, \mathbf{1}_{[0, t]}\right\rangle\right) ; \omega_{i} \in S^{\prime}(\mathbf{R})
$$

par un processus quelconque

$$
X_{t}(\omega)=\left(\left\langle\omega_{1}, \varphi_{t}^{1}\right\rangle, \cdots,\left\langle\omega_{d}, \varphi_{t}^{d}\right\rangle\right)
$$

avec $\varphi_{t}(u)=\left(\varphi_{t}^{1}, \cdots, \varphi_{t}^{d}\right) \in L^{2}(\mathbf{R}, d u)^{d} \forall t \geq 0$ et de plus pour tout $u$ de $\mathbf{R}$ l'application $t \longrightarrow \varphi_{t}(u)$ est mesurable. On pose:

$$
\begin{aligned}
& \left|\varphi_{t}\right|_{1}=\sum_{j=1}^{d}\left|\varphi_{t}^{j}\right|_{1}=\sum_{j=1}^{d} \int_{\mathbf{R}}\left|\varphi_{t}^{j}(u)\right| d u \\
& \left|\varphi_{t}\right|_{0}^{2}=\sum_{j=1}^{d}\left|\varphi_{t}^{j}\right|_{0}^{2}=\sum_{j=1}^{d} \int_{\mathbf{R}}\left|\varphi_{t}^{j}(u)\right|^{2} d u .
\end{aligned}
$$

On montre un résultat, voir (théorème 2) qui met en relation la dimension d de l'espace, et le nombre entier $\mathrm{N}$. Plus précisément on montre que si $\frac{\left|\varphi_{t}-\varphi_{s}\right|_{1}^{2 N}}{\left|\varphi_{t}-\varphi_{s}\right|_{0}^{d+2 N}} \in L^{1}\left(\Delta_{2}\right)$, l'intégral de Bochner $L^{(2 N)}=$ $\int_{\Delta_{2}} \delta^{(2 N)}\left(X_{t}-X_{s}\right) d t d s$ est une distribution de Hida i.e $L^{(2 N)}$ est un élément de $(S)^{*}$. 
Par ailleurs on démontre un résultat (théorème 3 ) analogue à celui de J.F.Le Gall [7] (corollaire 2.3 page 323) dans le cas du mouvement Brownien plan. Plus précisément on montre que $L_{x}=\int_{\Delta_{2}} \delta_{x}\left(B_{t}-\right.$ $\left.B_{s}\right) d t d s$ est une distribution de Hida pour $x \neq 0$ et que

$$
L_{x}-\frac{1}{\pi} \log \left(\frac{1}{|x|}\right)
$$

converge dans $(S)^{*}$ vers $\frac{1}{2 \pi}(\log 2-1-c)+L^{(2)}$ où c la constante d'Euler.

\section{Temps local d'intersection en analyse gaussi- enne}

\subsection{Elément d'analyse gaussienne}

On considère le triplet de Gelfand réel suivant:

$$
S(\mathbf{R})^{d} \hookrightarrow L^{2}(\mathbf{R})^{d} \hookrightarrow S^{\prime}(\mathbf{R})^{d}, d \geq 1
$$

$L^{2}(\mathbf{R})^{d}$ est muni du produit scalaire suivant:

$$
\langle f, g\rangle=\sum_{j=1}^{d} \int_{\mathbf{R}} f_{j}(u) g_{j}(u) d u
$$

La dualité, entre $S(\mathbf{R})^{d}$ et $S^{\prime}(\mathbf{R})^{d}$ est définie d'une façon naturelle par:

$$
\langle\omega, f\rangle=\sum_{j=1}^{d}\left\langle\omega_{j}, f_{j}\right\rangle
$$

où $\omega \in S^{\prime}(\mathbf{R})^{d}$ et $f \in S(\mathbf{R})^{d}$. On définit la mesure gaussienne $\gamma$ sur $S^{\prime}(\mathbf{R})^{d}$ par sa fonction caractéristique:

$$
C(f)=\int_{S^{\prime}(\mathbf{R})^{d}} e^{i\langle\omega, f\rangle} d \gamma(\omega)=e^{\frac{-\langle f, f\rangle}{2}}
$$

L'espace gaussien $\left(S^{\prime}(\mathbf{R})^{d}, \gamma\right)$ est dit espace de bruit blanc multidimensionnel. On obtient ainsi l'espace de Hilbert complexe suivant: 
$\left(L^{2}\right)=L^{2}\left(S^{\prime}(\mathbf{R})^{d}, d \gamma\right)$ qui est isomorphe par l'isométrie de Wiener-ItôSegal [4][8], à l'espace de Fock symétrique:

$$
\left(L^{2}\right) \simeq\left(\operatorname{Fock}\left(L^{2}(\mathbf{R}, d t)\right)\right)^{\otimes d} \simeq\left(\bigoplus_{n=0}^{+\infty} \operatorname{sym} L^{2}\left(\mathbf{R}^{k}, k ! d^{k} t\right)\right)^{\otimes d}
$$

Grâce à cette isométrie on montre que tout élément $\varphi \in\left(L^{2}\right)$ admet un développement en chaos de Wiener:

$$
\varphi(\omega)=\sum_{\vec{n}=0}^{+\infty}\left\langle: \omega^{\otimes \vec{n}}:, F_{\vec{n}}\right\rangle
$$

avec $F=\left\{F_{\vec{n}} ; \vec{n}=\left(n_{1}, \cdots, n_{d}\right) \in \mathbb{I}^{d}\right\} \in\left(\operatorname{Fock}\left(L^{2}(\mathbf{R}, d t)\right)\right)^{\otimes d}$, appelé noyau de $\varphi$ et pour tout $f=\left(f_{j}\right)_{1 \leq j \leq d} \in S(\mathbb{R})^{d}$

$$
\begin{aligned}
\left\langle: \omega^{\otimes \vec{n}}:, f^{\otimes \vec{n}}\right\rangle & =\prod_{j=1}^{d}\left\langle: \omega_{j}^{\otimes n_{j}}:, f_{j}^{\otimes n_{j}}\right\rangle \\
& =\prod_{j=1}^{d} \frac{\left|f_{j}\right|^{n_{j}}}{2^{\frac{n_{j}}{2}}} H_{n_{j}}\left\langle\omega_{j}, \frac{f_{j}}{\sqrt{2}\left|f_{j}\right|}\right\rangle
\end{aligned}
$$

où $\left(H_{n_{j}}\right)$ désignent les polynômes d'Hermite usuels.

On considère la suite $\left(A^{k}\right)_{k \geq 0}$, d'opérateurs de $L^{2}(\mathbf{R})^{d}$, où $A$ est défini par:

$$
A f=\left(A f_{j}\right)_{1 \leq j \leq d} \text { avec } A f_{j}=\left(-\frac{d^{2}}{d t^{2}}+t^{2}+1\right) f_{j}
$$

Puis en considèrant leur seconde quantification, on obtient une suite d'opérateurs sur l'espace $\left(\operatorname{Fock}\left(L^{2}(\mathbf{R}, d t)\right)\right)^{\otimes d}$ et enfin en utilisant l'isométrie (9) on obtient une suite $\left(\Gamma\left(A^{k}\right)\right)_{k \geq 0}$ d'opérateurs sur l'espace $\left(L^{2}\right)$.

On définit alors l'espace de fonctions test suivant:

$$
(S)=\operatorname{limproj}_{k \rightarrow+\infty}(S)_{k} \text { où }(S)_{k}=D\left(\Gamma\left(A^{k}\right)\right)
$$

Autrement dit, un élément $\varphi$ de $(S)$ vérifie:

$$
\varphi(\omega)=\sum_{\vec{n}=0}^{+\infty}\left\langle: \omega^{\otimes \vec{n}}:, F_{\vec{n}}\right\rangle
$$


et pour tout $p \geq 0$,

$$
\sum_{\vec{n}=0}^{+\infty} \vec{n} !\left|F_{\vec{n}}\right|_{p}^{2}<\infty
$$

On montre que l'espace $(S)$ ainsi construit est un espace nucléaire réflexif et par suite:

$$
(S)^{*} \text { fort }=\operatorname{limind}_{k \rightarrow+\infty}(S)_{-k}
$$

Les éléments de $(S)^{*}$ sont appelés distributions gaussiennes ou distributions de Hida. Ces espaces ont été introduits par Hida dans [4] et ont été étudiés, caractérisés et généralisés par de nombreux auteurs, $[11][9][8][6] \cdots$

Tout élément $\phi \in(S)^{*}$ s'écrit comme une série formelle de chaos de Wiener:

$$
\phi(\omega)=\sum_{\vec{n}=0}^{+\infty}\left\langle: \omega^{\otimes \vec{n}}:, \phi_{\vec{n}}\right\rangle
$$

où les $\left(\phi_{\vec{n}}\right)$ sont des noyaux distributions tempérées, et la dualité entre $(S)^{*}$ et $(S)$ notée $\langle\langle.,\rangle$.$\rangle prolonge le produit scalaire de \left(L^{2}\right)$ :

$$
\langle\langle\phi, \varphi\rangle\rangle=\sum_{\vec{n}=0}^{+\infty} \vec{n} !\left\langle\phi_{\vec{n}}, \varphi_{\vec{n}}\right\rangle
$$

où $\varphi$ est donnée par (10) et $\phi$ par (12). On caractérise les fonctionnelles du bruit blanc par leurs actions sur les exponentielles de Wick notées $: e^{\langle\omega, f\rangle}:$ et qui sont données par:

$$
\begin{aligned}
: e^{\langle\omega, f\rangle}: & \equiv \sum_{\vec{n}=0}^{+\infty} \frac{1}{\vec{n} !}\left\langle: \omega^{\otimes \vec{n}}:, f^{\otimes \vec{n}}\right\rangle \\
& =e^{\frac{-1}{2}\langle f, f\rangle} e^{\langle\omega, f\rangle}
\end{aligned}
$$

Définition 1. Soit $\phi=\sum_{\vec{n}=0}^{+\infty}\left\langle: \omega^{\otimes \vec{n}}: \phi_{\vec{n}}\right\rangle$ un élément de $(S)^{*}$

i) On définit la fonction de $S(\mathbf{R})^{d}$ par:

$$
S(\phi)(f)=\left\langle\left\langle\phi,: e^{\langle\cdot, f\rangle}:\right\rangle\right\rangle=\sum_{\vec{n}}\left\langle\phi_{\vec{n}}, f^{\otimes \vec{n}}\right\rangle
$$

et la transformation qui à $\phi$ associe $S \phi$ est dite la "S-transform" ou transformation chaotique de $\phi$. 
ii) Soit $k$ un entier $\geq 1$, on définit $\phi^{(k)} \in(S)^{*}$ par:

$$
\phi^{(k)}(\omega)=\sum_{\vec{n} ; n \geq k}\left\langle: \omega^{\otimes \vec{n}}:, \phi_{\vec{n}}\right\rangle
$$

où $n=|\vec{n}|$ est la longueur de $\vec{n}$.

Définition 2. Une fonction $G: S(\mathbf{R})^{d} \longrightarrow \mathrm{C}$ est dite $U$-fonctionnelle si:

i) $G\left(\lambda f_{1}+f_{2}\right)$ est entière en $\lambda$ pour toute $f_{1}, f_{2} \in S(\mathbf{R})^{d}$.

ii) Il existe $c_{1}, c_{2}>0$ et $p \geq 0$ tel que $|G(f)| \leq c_{1} e^{c_{2}\left|A^{p} f\right|_{0}^{2}}, \quad \forall f \in$ $S(\mathbf{R})^{d}$.

Théorème 1 (de caractérisation). Les deux assertions suivantes sont équivalentes:

i) $G$ est une $U$-fonctionnelle.

ii) $G$ est la $S$-transformée d'une distribution gaussienne $\phi$ élément de $(S)^{*}$.

Remarque. Le théorème précédant a été démontré dans le cas $d=1$ dans [11] et dans un cadre beaucoup plus général dans [9]. Le corollaire suivant (voir [1] corollary 1, [14]), constitue l'outil fondamental de ce travail.

Corollaire 1. Soit $(\Omega, \mathcal{B}, m)$ un espace mesuré et

$$
\begin{aligned}
\phi: & \longrightarrow(S)^{*} \\
\lambda & \longrightarrow \phi_{\lambda}
\end{aligned}
$$

On suppose que la transformée chaotique de $\phi_{\lambda}, S \phi_{\lambda}$ vérifie:

i) $S \phi_{\lambda}(f)$ est mesurable en $\lambda$, pour toute $f \in S(\mathbf{R})^{d}$.

ii) Il existe $p>0$ indépendant de $\lambda$, deux fonctions $c_{1}$ et $c_{2}$ de $\lambda$ avec $c_{1} \in L^{1}(\Omega), c_{2} \in L^{\infty}(\Omega)$ tel que:

$$
\mid S \phi_{\lambda}(f) \leq c_{1}(\lambda) e^{c_{2}(\lambda)\left|A^{p} f\right|_{0}^{2}} \forall f \in S(\mathbf{R})^{d}
$$


alors $\phi$ est intégrable au sens de Bochner, dans un certain $(S)_{-q}$ i.e

$$
\int_{\Omega} \phi_{\lambda} d \lambda \in(S)^{*}
$$

et

$$
S\left\{\int_{\Omega} \phi_{\lambda} d \lambda\right\}(f)=\int_{\Omega} S \phi_{\lambda}(f) d \lambda
$$

\subsection{Application au temps local d'intersection}

Soit $t>0$, la fonction $\mathbf{1}_{[o, t]}$ est un élément de $L^{2}(\mathbf{R}, d u)$ donc on peut l'approcher par une suite de fonctions de $S(\mathrm{R})$, ceci permet de définir le mouvement Brownien comme fonctionnelle du bruit blanc, $\left(S^{\prime}(\mathbf{R}), \gamma\right)$ ( $\gamma$ étant la mesure gaussienne standard), $B_{t}=\left\langle\omega, \mathbf{1}_{[o, t]}\right\rangle, \forall \omega \in S^{\prime}(\mathbf{R})$ $([4][8])$.

En utilisant le fait que la transformée de Fourier de $\delta, \mathcal{F}(\delta)(\xi)=1$, on obtient formellement l'égalité suivante:

$$
\delta\left(B_{t}-B_{s}\right)=\delta\left(\left\langle\omega, 1_{[s, t]}\right\rangle\right)=\frac{1}{2 \pi} \int_{\mathbf{R}} e^{i\left\langle\omega, \mathbf{1}_{[s, t]}\right\rangle \xi} d \xi
$$

Ainsi en regardant l'intégrale formelle figurant dans (15) comme étant une intégrale de Bochner, il est possible de donner un sens à $\delta\left(B_{t}-B_{s}\right)$ comme une distribution gaussienne.

On va donc dans la suite étendre les résultats obtenus dans [1] à des processus plus généraux que celui du mouvement Brownien.

On définit pour cela la famille de fonctionnelles du bruit blanc à valeur vectorielle:

$$
X_{t}(\omega)=\left(\left\langle\omega_{1}, \varphi_{t}^{1}\right\rangle, \ldots,\left\langle\omega_{d}, \varphi_{t}^{d}\right\rangle\right)=(\omega, \varphi)
$$

où $\varphi_{t}=\left(\varphi_{t}^{1}, \cdots, \varphi_{t}^{d}\right) \in L^{2}(\mathbf{R}, d u)^{d}, \forall t \geq 0$ et tel que pour tout $u \in \mathbf{R}$ l'application: $t \longrightarrow \varphi_{t}(u)$ est mesurable.

\section{Exemples.}

1. Si $\varphi_{t}^{i}=\mathbf{1}_{[0, t]}, \forall i=1, \ldots, d$ alors $\left\{X_{t}\right\}$ est un mouvement Brownien multidimensionnel. 
2. Si $\varphi_{t}^{i}=e^{-\lambda t} \mathbf{1}_{-\infty, t]}(u) e^{\lambda u}, \forall i=1, \ldots, d$ et $\lambda>0$ alors $\left\{X_{t}\right\}$ est le processus d'Ornstein-Uhlenbeck de parametre $\lambda$.

Proposition 1. L'intégral de Bochner:

$$
\delta\left(X_{t}-X_{s}\right)=\frac{1}{(2 \pi)^{d}} \int_{\mathbf{R}^{d}} e^{i \xi\left(X_{t}-X_{s}\right)} d \xi
$$

est un élément de $(S)^{*}$ lorsque $\left|\varphi_{t}-\varphi_{s}\right|_{0} \neq 0$, de plus

$$
S\left(\delta\left(X_{t}-X_{s}\right)\right)(f)=\frac{1}{2 \pi^{\frac{d}{2}}} \frac{1}{\left|\varphi_{t}-\varphi_{s}\right|_{0}^{d}} \exp \left(\frac{-1}{2} \frac{\left(f, \varphi_{t}-\varphi_{s}\right)_{0}^{2}}{\left|\varphi_{t}-\varphi_{s}\right|_{0}^{2}}\right)
$$

avec

$$
\left(f, \varphi_{t}-\varphi_{s}\right)_{0}^{2}=\sum_{j=1}^{d}\left\{\int_{\mathbf{R}} f_{j}(u)\left(\varphi_{t}^{j}-\varphi_{s}^{j}\right)(u) d u\right\}^{2}
$$

Démonstration. On commence par calculer la transformée chaotique de la fonctionnelle du bruit blanc $e^{i \xi\left(X_{t}-X_{s}\right)}$

$$
\begin{aligned}
S\left(e^{i \xi\left(X_{t}-X_{s}\right)}\right)(f) & =S\left(\prod_{j=1}^{d} e^{i \xi_{j}\left(\omega_{j}, \varphi_{t}^{j}-\varphi_{s}^{j}\right\rangle}\right)(f) \\
& =e^{\frac{-1}{2} \xi^{2}\left|\varphi_{s}-\varphi_{t}\right|_{0}^{2}} e^{i \xi\left(f, \varphi_{t}-\varphi_{s}\right)_{0}}
\end{aligned}
$$

or $\left|\xi\left(f, \varphi_{t}-\varphi_{s}\right)\right| \leq \frac{1}{4} \xi^{2}\left|\varphi_{t}-\varphi_{s}\right|_{0}^{2}+\frac{\left(f, \varphi_{t}-\varphi_{s}\right)_{0}^{2}}{\left|\varphi_{t}-\varphi_{s}\right|_{0}^{2}}$, on obtient donc

$$
\left|S\left(e^{i \xi\left(X_{t}-X_{s}\right)}\right)(f)\right| \leq e^{-\frac{1}{4} \xi^{2}\left|\varphi_{t}-\varphi_{s}\right|_{0}^{2}} e^{\frac{\left(f, \varphi_{t}-\varphi_{s}\right)_{0}^{2}}{\left|\varphi_{t}-\varphi_{s}\right|_{0}^{2}}}
$$

l'application

$$
\xi \longrightarrow e^{-\frac{1}{4} \xi^{2}\left|\varphi_{t}-\varphi_{s}\right|_{0}^{2}} \in L^{1}\left(\mathbf{R}^{d}, d \xi\right)
$$

$\operatorname{pour} \varphi_{t} \neq \varphi_{s}$

$$
\begin{aligned}
\left(f, \varphi_{t}-\varphi_{s}\right)_{0}^{2} & =\sum_{j=1}^{d}\left\{\int_{\mathbf{R}} f_{j}(u)\left(\varphi_{t}^{j}-\varphi_{s}^{j}\right)(u) d u\right\}^{2} \\
& \leq\left|\varphi_{t}-\varphi_{s}\right|_{0}^{2} \sum_{j=1}^{d}\left|f_{j}\right|_{0}^{2} \\
& =\left|\varphi_{t}-\varphi_{s}\right|_{0}^{2}|f|_{0}^{2}
\end{aligned}
$$


finalement on obtient

$$
\left|S\left(e^{i \xi\left(X_{t}-X_{s}\right)}\right)(f)\right| \leq e^{-\frac{1}{4} \xi^{2}\left|\varphi_{t}-\varphi_{s}\right|_{0}^{2}} e^{|f|_{0}^{2}}
$$

ce qui entraine, d'après le corollaire 1 que $\delta\left(X_{t}-X_{s}\right) \in(S)^{*}$

$$
\begin{aligned}
S\left(\delta\left(X_{t}-X_{s}\right)\right)(f) & =\frac{1}{(2 \pi)^{d}} \int_{\mathbf{R}^{d}} S\left(e^{i \xi\left(X_{t}-X_{s}\right)}\right)(f) d \xi \\
& =\prod_{j=1}^{d} \frac{1}{2 \pi} \int_{\mathbf{R}} e^{-\frac{1}{2} \xi_{j}^{2}\left|\varphi_{t}-\varphi_{s}\right|_{0}^{2}+i\left\langle f_{j}, \varphi_{t}^{j}-\varphi_{s}^{j}\right\rangle_{0}} d \xi_{j} \\
& =\prod_{j=1}^{d} \frac{1}{\sqrt{2 \pi}}\left|\varphi_{t}-\varphi_{s}\right|_{0}^{-1} \exp \left(\frac{-1}{2} \frac{\left\langle f_{j}, \varphi_{t}^{j}-\varphi_{s}^{j}\right\rangle_{0}^{2}}{\left|\varphi_{t}-\varphi_{s}\right|_{0}^{2}}\right) \\
& =\frac{1}{(2 \pi)^{d / 2}} \frac{1}{\left|\varphi_{t}-\varphi_{s}\right|_{0}^{d}} \exp \left(\frac{-1}{2} \frac{\left(f, \varphi_{t}-\varphi_{s}\right)_{0}^{2}}{\left|\varphi_{t}-\varphi_{s}\right|_{0}^{2}}\right)
\end{aligned}
$$

Une fois $\delta\left(X_{t}-X_{s}\right)$ bien définie, on veut définir:

$$
L=\int_{\Delta_{2}} \delta\left(X_{t}-X_{s}\right) d s d t
$$

comme une fonctionnelle du bruit blanc, or

$$
\mathbb{E}(L)=\int_{\Delta_{2}} S\left(\delta\left(X_{t}-X_{s}\right)\right)(0) d s d t=\int_{\Delta_{2}} \frac{d s d t}{(2 \pi)^{d / 2}\left|\varphi_{t}-\varphi_{s}\right|_{0}^{d}}=+\infty
$$

pour les deux cas cités dans l'exemple ci dessus et pour $d \geq 2$. Donc $L \notin(S)^{*}$, par suite on étudie la renormalisation $L^{(2 N)}, N \geq 1$ (définiton 1.ii)) de $L$. Ce théorème exprime la relation entre $N$ et la dimention $d$.

Théorème 2. Soit $X_{t}(\omega)=\left(\left\langle\omega_{1}, \varphi_{t}^{1}\right\rangle, \ldots,\left\langle\omega_{d}, \varphi_{t}^{d}\right\rangle\right)$ tel que $\varphi_{t}=$ $\left(\varphi_{t}^{1}, \cdots, \varphi_{t}^{d}\right) \in L^{2}(\mathbf{R}, d u)^{d} \forall t \geq 0$ et $\forall u \in \mathbf{R}$ l'application: $t \longrightarrow \varphi_{t}(u)$ est mesurable.

Si de plus

$$
\frac{\left|\varphi_{t}-\varphi_{s}\right|_{1}^{2 N}}{\left|\varphi_{t}-\varphi_{s}\right|_{0}^{d+2 N}} \in L^{1}\left(\Delta_{2}\right)
$$

alors

$$
L^{(2 N)}=\int_{\Delta_{2}} \delta^{(2 N)}\left(X_{t}-X_{s}\right) d s d t
$$


est un élément de $(S)^{*}$.

Où $\delta^{(2 N)}\left(X_{t}-X_{s}\right)$ est la distribution de Hida définie en appliquant la définition 1.ii) à $\delta\left(X_{t}-X_{s}\right)$.

Démonstration. D'après la proposition 1 on a:

$$
\begin{gathered}
S\left(\delta\left(X_{t}-X_{s}\right)\right)(f)=\frac{1}{(2 \pi)^{\frac{d}{2}}} \frac{1}{\left|\varphi_{t}-\varphi_{s}\right|_{0}^{d}} e^{-\frac{1}{2} \frac{\left(f, \varphi_{t}-\varphi_{s}\right)_{0}^{2}}{\left|\varphi_{t}-\varphi_{s}\right|_{0}^{2}}} \\
=\frac{1}{(2 \pi)^{d / 2}} \frac{1}{\left|\varphi_{t}-\varphi_{s}\right|_{0}^{d}} \sum_{\vec{n}=0}^{+\infty} \frac{1}{\vec{n} !}\left(\frac{-1}{2\left|\varphi_{t}-\varphi_{s}\right|_{0}^{2}}\right)^{n}\left(f, \varphi_{t}-\varphi_{s}\right)_{0}^{2 \vec{n}} \\
\frac{1}{(2 \pi)^{d / 2}} \frac{1}{\left|\varphi_{t}-\delta_{s}\right|_{0}^{d}} \sum_{\vec{n} ; n \geq N} \frac{1}{\vec{n} !}\left(\frac{-1}{2\left|\varphi_{t}-\varphi_{s}\right|_{0}^{2}}\right)^{n}\left(f, \varphi_{t}-\varphi_{s}\right)_{0}^{2 \vec{n}} \\
\left(f, \varphi_{s}-\varphi_{s}\right)_{0}^{2 \vec{n}}=\prod_{j=1}^{n} \int_{\mathbf{R}} f_{j}(u)\left(\varphi_{t}^{j}-\varphi_{s}^{j}\right)(u) d u \\
\left|\left(f, \varphi_{t}-\varphi_{s}\right)_{0}^{2 \vec{n}}\right| \leq \prod_{j=1}^{d} \sup _{u}\left|f_{j}(u)\right|^{2 n_{j}}\left|\varphi_{t}^{j}-\varphi_{s}^{j}\right|_{1}^{2 n_{j}} \\
\leq\left|\varphi_{t}-\varphi_{s}\right|_{1}^{2 n} \prod_{j=1}^{d}\left(\sup _{n \in \mathbf{R}}\left|f_{j}(n)\right|\right)^{2 n_{j}}
\end{gathered}
$$

on obtient donc

$$
\begin{gathered}
\left|S\left(\delta^{2 N}\left(X_{t}-X_{s}\right)\right)(f)\right| \leq \\
\frac{1}{(2 \pi)^{\frac{d}{2}}} \frac{1}{\left|\varphi_{t}-\varphi_{s}\right|_{0}^{d}} \sum_{\vec{n}, n \geq N} \frac{1}{\vec{n} !}\left(\frac{\left|\varphi_{t}-\varphi_{s}\right|_{1}^{2}}{2\left|\varphi_{t}-\varphi_{s}\right|_{0}^{2}}\right)^{n} \prod_{j=1}^{d}\left(s u p_{u}\left|f_{j}\right|\right)^{2 n_{j}} \\
\leq \frac{1}{(2 \pi)^{\frac{d}{2}}} \frac{\left|\varphi_{t}-\varphi_{s}\right|_{1}^{2 N}}{\left|\varphi_{t}-\varphi_{s}\right|_{0}^{d+2 N}} e^{\frac{1}{2} \sum_{j=1}^{d}\left|f_{j}\right|_{\infty}^{2}}
\end{gathered}
$$

Alors comme

$$
\frac{\left|\varphi_{t}-\varphi_{s}\right|_{1}^{2 N}}{\left|\varphi_{t}-\varphi_{s}\right|_{0}^{d+2 N}} \in L^{1}\left(\Delta_{2}\right)
$$


on aura

$$
L^{(2 N)}=\int_{\Delta_{2}} \delta^{(2 N)}\left(X_{t}-X_{s}\right) d s d t \in(S)^{*}
$$

\section{Corollaire 2.}

1. Si $\left\{X_{t}\right\}$ est le processus du mouvement Brownien $\left\{B_{t}\right\}$ alors:

$$
L^{(2 N)}=\int_{\Delta_{2}} \delta^{(2 N)}\left(B_{t}-B_{s}\right) d s d t
$$

est un élément de $(S)^{*}$ pour $2 N>d-2$.

2. Si $\left\{X_{t}\right\}$ est le processus d'Ornstein-Uhlenbeck:

$$
L^{(2 N)}=\int_{\Delta_{2}} \delta^{(2 N)}\left(X_{t}-X_{s}\right) d s d t
$$

est un élément de $(S)^{*}$ pour $2 N>d-2$.

\section{Démonstration.}

1. Si $X_{t}=B_{t}$ alors $\varphi_{t}=\left(1_{[0, t]}, \cdots, \mathbf{1}_{[0, t]}\right)$ donc $\left|\varphi_{t}-\varphi_{s}\right|_{0}=\sqrt{d|t-s|}$ et $\left|\varphi_{t}-\varphi_{s}\right|_{1}=d|t-s|$ par suite

$$
\frac{\left|\varphi_{t}-\varphi_{s}\right|_{1}^{2 N}}{\left|\varphi_{t}-\varphi_{s}\right|_{0}^{d+2 N}}=(d|t-s|)^{N-\frac{d}{2}} \in L^{1}\left(\Delta_{2}\right)
$$

pour $2 N>d-2$, (on retrouve ainsi le théorème 2 de [1]).

2. Si $\left\{X_{t}\right\}$ est le processus d'Ornstein-Uhlenbeck alors

$$
\begin{aligned}
& \varphi_{t}(u)=e^{-\lambda(t-u)}\left(\mathbf{1}_{]-\infty, t]}, \cdots, \mathbf{1}_{]-\infty, t]}\right), \lambda>0 \text { donc } \\
& \left|\varphi_{t}^{j}-\varphi_{s}^{j}\right|_{1}=\frac{2}{\lambda}\left(1-e^{-\lambda(t-s)}\right) \simeq 2|t-s| \forall j=1, \cdots, d \\
& \left|\varphi_{t}-\varphi_{s}\right|_{1} \simeq 2 d|t-s| \\
& \left|\varphi_{t}^{j}-\varphi_{s}^{j}\right|_{0}=\left[\frac{e^{\lambda(t-s)}+1-e^{-2 \lambda(t-s)}}{2 \lambda}\right]^{\frac{1}{2}} \simeq \frac{|t-s|^{\frac{1}{2}}}{2} \forall j=1, \ldots, d \\
& \left|\varphi_{t}-\varphi_{s}\right|_{0} \simeq \frac{(d|t-s|)^{\frac{1}{2}}}{2} \text { et par suite } \\
& \frac{\left|\varphi_{t}-\varphi_{s}\right|_{1}^{2 N} \simeq 2^{4 N+d} d^{N-\frac{d}{2}}|t-s|^{N-\frac{d}{2}} \in L^{1}\left(\Delta_{2}\right) \text { pour } 2 N>d-2}{\left|\varphi_{t}-\varphi_{s}\right|_{0}^{2 N+d}}
\end{aligned}
$$




\section{Remarques.}

1) Il y a une ressemblance entre les résultats déjà obtenus sur le temps local d'intersection et nos résultats, en effet lorsque $d=1,2 N>-1$ et donc $N=0$ par suite $L=\int_{0}^{1} \int_{0}^{1} \delta\left(X_{t}-X_{s}\right) d s d t \in(S)^{*}$, ceci rejoint les résultats de [3][5].

2) Si $d=2$, donc $N=1$ et par suite il suffit de retrancher l'espérance, ce qui rejoint parfaitement la renormalisation de Varadhan [13][7].

On va maintenant, démontrer un résultat analogue à celui de [7] corollaire 2.4 dans le cas où le processus $\left\{X_{t}\right\}$ est le processus du mouvement Brownien plan $\left\{B_{t}\right\}$. Pour cela, on commence par donner deux propositions qui restent vraies pour $d$ quelconque.

Proposition 2. Soit $x \in \mathbf{R}^{d}$, on désigne par $\delta_{x}$ la mesure de Dirac de $\mathbf{R}^{d}$ en $x$, alors

$$
\delta_{x}\left(B_{t}-B_{s}\right)=\frac{1}{(2 \pi)^{d}} \int_{\mathbf{R}^{d}} e^{i \xi\left(B_{t}-B_{s}\right)} e^{-i x \xi} d \xi \in(S)^{*}
$$

de plus

$$
S\left(\delta_{x}\left(B_{t}-B_{s}\right)\right)(f)=\frac{1}{(2 \pi|t-s|)^{\frac{d}{2}}} \exp \left\{\frac{-x^{2}}{|t-s|}+x \frac{\int_{s}^{t} f d u}{|t-s|}-\frac{\left(\int_{s}^{t} f d u\right)^{2}}{2|t-s|}\right\} .
$$

Proposition 3. Soit $x \in \mathbf{R}^{d}-\{0\}$, alors

$$
L_{x}=\int_{\Delta_{2}} \delta_{x}\left(B_{t}-B_{s}\right) d s d t \in(S)^{*}
$$

et

$$
\mathbb{E}\left(L_{x}\right)=\int_{\Delta_{2}} \frac{e^{-\frac{x^{2}}{2|t-s|}}}{(2 \pi|t-s|)^{\frac{d}{2}}} d s d t
$$

On suppose maintenant que $d=2$.

Théorème 3 .

$$
L_{x}-\frac{1}{\pi} \log \left(\frac{1}{|x|}\right)
$$


converge dans $(S)^{*}$ vers

$$
\frac{1}{2 \pi}(\log 2-1-c)+L^{(2)}
$$

où c est la constante d'Euler.

Démonstration. On montre d'abord que

$$
\delta_{x}\left(B_{t}-B_{s}\right)-\frac{e^{\frac{-x^{2}}{2|t-s|}}}{2 \pi|t-s|}
$$

converge dans $(S)^{*}$ vers $\delta^{(2)}\left(B_{t}-B_{s}\right)$, en effet :

$$
S\left(\delta_{x}\left(B_{t}-B_{s}\right)\right)(f)-\frac{e^{\frac{-x^{2}}{2|t-s|}}}{2 \pi|t-s|}
$$

converge quand $x \longrightarrow 0$ vers

$$
\frac{1}{2 \pi|t-s|}\left\{e^{-\frac{\left(\int_{s}^{t} f d u\right)^{2}}{2|t-s|}}-1\right\}
$$

qui n'est autre que la transformée $\mathrm{S}$ de $\delta^{(2)}\left(B_{t}-B_{s}\right)$. Ce qui implique en utilisant le corollaire 1 et le théorème de convergence dominée pour les intégrales de Bochner que:

$$
L_{x}-\int_{\Delta_{2}} \frac{e^{\frac{-x^{2}}{2|t-s|}}}{2 \pi|t-s|} d s d t
$$

converge dans $(S)^{*}$ vers $L^{(2)}$. Pour finir la démonstration du théorème 3 , on utilise le lemme technique suivant:

Lemme 1. [15]

$$
\int_{\Delta_{2}} \frac{e^{\frac{-x^{2}}{2|t-s|}}}{2 \pi|t-s|} d s d t-\frac{1}{\pi} \log \frac{1}{|x|} \longrightarrow \frac{1}{2 \pi}(\log 2-1-c)
$$

quand $x$ tend vers zéro, où c est la constante d'Euler.

Remerciement. Les auteurs remercient le Professeur Ludwig Streit de l'université de Bielefeld, pour les très fructueuses discussions qu'ils ont eues avec lui lors de son passage à Tunis en Septembre 1996 et en septembre 1997. C'est en réponse à l'une de ses questions que ce papier a été élaboré. 


\section{References}

[1] M. Faria, T. Hida, L. Streit, H. Watanabe, Intersection local time as Generalized White Noise Functionals.(Preprint) 1996.

[2] D. Geman, J. Horowitz, J. Rosen, A local time Analysis of intersections of Brownian paths in the plane. The Ann. Prob. 12, N 1, 86-107 (1984).

[3] D. Geman, J. Horowitz, Occupation densities. The Aannals of Proba 1980, vol 8, $N 1,1-67$.

[4] T. Hida, "Brownian Motion", Springer, Berlin/Heidelberg/ New york, 1980.

[5] K. Itô and H.P. Mckean, Diffusion Processes and Their Sample Paths. Academic Press, New York, (1965).

[6] Yu. Kontratiev, P. Leukert, J. Potthoff, L. Streit, W. Westerkamp, Generalized functionals in Gaussian spaces, the characterization theorem revisted. J.F.A Vol $141 n^{\circ} 2$ Page 301-318 (1996).

[7] J.F. Le Gall, Sur le temps local d'intersection du mouvement Brownien plan et la méthode de renormalisation de Varadhan. Sem de Prob. XIX, 1983/84. LNM 1123 (1985) 314-331. Springer, Berlin.

[8] N. Obata, White noise calculus and Fock space. LNM 1577 (1994).

[9] H. Ouerdiane, Fonctionnelles analytiques avec conditions de croissance et application à l'analyse gaussienne. Japanese Journal of Math. Vol 20, $N^{\circ} 1$. pp. 187-198.(1994).

[10] H. Ouerdiane, Noyaux et symboles d'opérateurs sur des fonctionnelles analytiques gaussiennes. Japanese Journal of Math. Vol 21. $N^{\circ} 1$. pp 223-234 Juin 1995.

[11] J. Potthoff, L. Streit, A characterization of Hida distributions. J.Func.Anal. 101 p 212-229 (1991).

[12] J. Rosen, Alocal time Approach to the self-intersections of Brownian Paths in space. Commun.Math.Phys. 88, 327-338 (1983).

[13] S.R.S. Varadhan, Appendix to "Euclidean quantum field theory" by K.Symanzik. In "local quantum theory" (R.J.st.ed). Academic new york (1969).

[14] H. Watanabe, The local time self-intersections of Brownian Motions as generalized Brownian functionals, Lett. Math. Phys. 23, 1-9. (1991).

[15] M. Yor, Renormalisation et convergence en loi pour les temps locaux d'intersection du movement Brownien Sem de Prob XIX 1983/84. LNM 1123 (1985) 350-365. Springer, Berlin. 
H.Ouerdiane et A.Rezgui

Faculté des Sciences de Tunis

Département de Mathématique

Campus universitaire 1060 Tunis

Tunisie.

E-mail: rezgui@physik.uni-bielefeld.de

E-mail: habib.ouerdiane@fst.rnu.tn

Recibido: 27 de Marzo de 1998

Revisado: 1 de Agosto de 2000 